\title{
Dynamic response of utility tunnel during the passage of Rayleigh waves*
}

\author{
$\mathrm{Jie} \mathrm{Li}^{1, \uparrow}$ Qingxia Yue ${ }^{2}$ and Jun Chen ${ }^{1}$ \\ ${ }^{1}$ State Key Laboratory for Disaster Reduction in Civil Engineering, Tongii University, Shanghai 200092, China \\ ${ }^{2}$ School of Civil Engineering, Shandong Jianzhu University, Ji'nan 250101, China
}

\begin{abstract}
The modeling methodologies and calculation of dynamic response of underground structure under Rayleigh waves is investigated in this paper. First the free field responses under Rayleigh waves are analyzed and the numerical results agree well with the theoretical results. Then, the approximate Rayleigh waves are put forward based on the preliminary research, and Rayleigh wave field is obtained through fast Fourier transform technique. Taking a utility tunnel as an example, its dynamic responses under Rayleigh waves is calculated by ABAQUS. The results demonstrate that bending deformation is the main component of structural deformation and the deformation at the top of the structure is about twice as much as that at bottom of the structure. The effect of soil-structure interface and the buried depth of underground structure are also investigated via parameter analysis. For the shallow buried underground structures, Rayleigh waves can be the key factor to control the responses and damage of the structure.
\end{abstract}

Key words: utility tunnel; Rayleigh wave; dynamic response; fast Fourier transform CLC number: P315.9 Document code: A

\section{Introduction}

Underground structures are becoming more and more prevalent in the modern world because of the decreasing availability of aboveground space and resources due to fast growing population. This investigation concentrates on utility tunnel, which is a kind of underground structure that holds many kinds of lifelines, such as wires, conduits and pipes of power, gas water supply and communication utility system. The first modern utility tunnel is built in Paris. Now Japan is the leading country in construction of utility tunnel. In China, the first modern utility tunnel was built in Shanghai in 1994 and there are two utility tunnels in Shanghai now. Figure 1 is the inside view of an as-built utility tunnel.

Due to its remarkable advantages in improving reliability, reducing deferred maintenance, utility tunnel is tending to be major choice for underground infrastructure construction in the future, not only for megalopolis

\footnotetext{
* Received 21 October 2009; accepted in revised form 15 January 2010; published 10 February 2010.

^Corresponding author. e-mail: lijie@tongji.edu.cn

(C) The Seismological Society of China and Springer-Verlag Berlin Heidelberg 2010
}

but also for small towns. However, there is still limited research to date on the seismic response analysis of the utility tunnel. Utility tunnel is typically box-shaped and shallow-buried concrete structures. Statistical data of damage in underground structures under strong seismic waves show that shallow tunnels are at higher risk during an earthquake than deeper tunnels; roughly $60 \%$ of the total cases with overburden depth less than $50 \mathrm{~m}$ suffered certain damages (Wang, 1993). It is thus reasonable to conclude that the utility tunnel may be damaged more severely than deep ones during an earthquake. So the shallow-buried underground structure should be paid more attention under earthquake excitations.

Majority of the researches conducted on the dynamic response of underground structures concentrates on the study of soil-structure interaction. Most of these researches assume vertically propagating shear waves (S-waves). A comprehensive review on this subject has been presented by Hashash et al (2001). This assumption has been employed because of some considerable computational difficulties in exact prediction of responses from a potential earthquake. Moreover, it is known that for motions close to the epicenter, continuous transmission of upward propagating S-wave through 
increasingly soft soil layers gradually changes their direction. Different methods have been used to investigate the dynamic soil-structure interaction, such as pseudostatic method (Wang, 1993; Penzien and Wu, 1998; Penzien, 2000; Huo, 2005), numerical method (Matsui et al, 2001; Huo, 2005), and experimental method (Prasad et al, 2004).

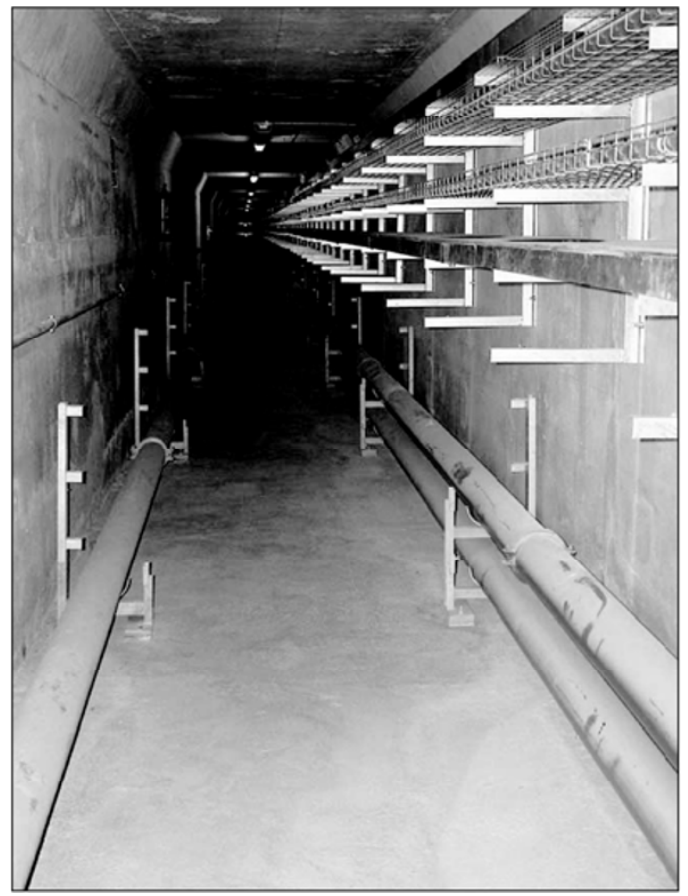

Figure 1 Photo of an existing utility tunnel.

Despite of the significant progress in understanding behavior of underground structures under seismic excitation, very little is known about their response, especially when the soil is excited under Rayleigh waves. Miller and Pursey (1955) studied the partition of energy between elastic waves in a semi-infinite solid, which investigated the power of three waves contributing to total earthquake input respectively; the result revealed that Rayleigh wave is $67.3 \%$, the shear wave is $25.8 \%$ and the compression wave is $6.9 \%$. Furthermore, surface waves induce motions and stresses in the soil and structure which are substantially different from those calculated based on the assumption of vertical S-waves. Makris (1994) and Makris and Badoni (1995) studied the response of pile during the passage of Rayleigh waves. It is well established that surface waves have a significant contribution to response of the shallow-buried structures. The above reasons motivate the study reported herein.

This paper studies the dynamic response of utility tunnel during the passage of Rayleigh waves. First, the free field analysis is conducted using FEM of wave motion involved in the calculation. The results are compared with analytical solutions. Based on fast Fourier transform technique (FFT), approximate Rayleigh wave field is obtained. Then the dynamic response of utility tunnel during Rayleigh waves is calculated and some factors such as interface condition and the buried depth are analyzed.

\section{Finite element formulation of wave motion}

Based on fundamental theory of finite element method, the computational region is defined as a discrete system and the dynamic motion equation can be written as

$$
M \ddot{u}+C \dot{u}+K u=P,
$$

in which $\boldsymbol{M}, \boldsymbol{C}$ and $\boldsymbol{K}$ are mass, damping and stiffness matrices, respectively. $\ddot{\boldsymbol{u}}, \dot{\boldsymbol{u}}$ and $\boldsymbol{u}$ are acceleration, velocity and displacement vectors. $\boldsymbol{P}$ is excitation vector, which are deterministic or stochastic. From formula (1), we can see that the finite element formulation of wave motion is the same as common finite element formulation. The size of element and time increment should be controlled in order to reduce the numerical error due to the discrete system instead of the continuous one.

A unique characteristic of finite element simulation technique of wave motion is that an artificial boundary condition (ABC) should be employed to model the energy radiation phenomena of the computational region in infinite media. Many kinds of ABCs have been put forward to reduce spurious energy reflection. Alterman and Kara (1968) first put forward the concept of placing the artificial truncated boundary far away from the domain which we are interested in. The most widely used is the viscous boundary (Lysmer and Kuhlemeyer, 1969), which replaces the far field with viscous damping. In order to simulate the elastic resilience of soil, the viscous-spring boundary, which adds spring at the boundary simultaneously, is also used widely at present. Clayton and Engquist (1977) deduced a paraxial approximation boundary based on the elastic wave propagation equation, which is the remarkable achievement in the 1970s about the artificial boundary condition. Liao et al (1984) proposed a more general used multi-transmitting boundary condition from the general wave propagation equation.

The infinite element is also put forward to deal with the infinite domain, which uses the concept of the 
mapping function to model the infinite domain. In ABAQUS, first- and second-order infinite elements are provided that are based on the work of Zienkiewicz et al (1981) for static response and of Lysmer and Kuhlemeyer (1969) for dynamic response. The elements are used in conjunction with traditional finite elements, which model the area around the region of interest, with the infinite elements modeling the far-field region. The dynamic response of the infinite elements is based on the consideration of plane body waves traveling orthogonally to the boundary. We choose the proper damping constants $d_{\mathrm{P}}$ and $d_{\mathrm{S}}$ to eliminate reflection of longitudinal and shear wave energy back into the medium respectively, in which $d_{\mathrm{P}}=\rho c_{\mathrm{P}}, d_{\mathrm{S}}=\rho c_{\mathrm{S}}, \rho$ is the density of the medium and $c_{\mathrm{P}}, c_{\mathrm{S}}$ are velocity of the longitudinal and shear wave respectively. These values of boundary damping are added into the infinite elements in ABAQUS. It has been verified that the infinite elements transmit all normally impinging plane body waves exactly provided that the material behavior close to the boundary is linearly elastic.

General problems involve non plane body waves that do not impinge on the boundary from an orthogonal direction and may also involve Rayleigh surface waves and Love waves. Nevertheless, these "quiet" boundaries work quite well even for such general cases, provided that they are arranged so that the dominant direction of wave propagation is orthogonal to the boundary or, at free surfaces and interfaces where Rayleigh or Love waves are of concern, they are orthogonal to the surface. As the boundaries are "quiet" rather than silent (perfect transmitters of all waveforms), and because the boundaries relying on the solution adjacent to them are linearly elastic, they should be placed at some reasonable distance from the region of main interest. Ismail and Mullen (2000) and Yue et al (2006) made some comparisons about the dashpot boundary and the infinite element in ABAQUS. The results showed that the infinite element in ABAQUS have many advantages over the dashpot/viscous boundary.

On the other hand, the discretization of the computational region will result in some undesirable phenomena, such as dispersion, parasitic oscillation, and anisotropy of wave motion. However, all these numerical problems can be limited to an acceptable extent by choosing a small discretization mesh size $\Delta x$ so that the frequency range of the traveling waves is much less than Nyquist frequency. According to previous researches (Liao, 2002; Lysmer and Kuhlemeyer, 1969; Smith,
1974), the element mesh size $\Delta x$ should be less than $1 / 8-1 / 12$ of the minimum wavelength $\lambda_{\min }$ to guarantee the accuracy. Thus, the maximum frequency for a mesh discretization scheme is

$$
\omega_{\max }=\frac{2 c_{\min } \pi}{(8-12) \Delta x},
$$

in which $c_{\min }$ is the minimum wave velocity corresponding to soil media. In order to increase the maximum frequency of a computational zone, the mesh size should be decreased, but the computational efforts will increase. In practical analysis, a trade-off between the maximum frequency and the mesh size has to be achieved.

\section{Soil displacement due to Rayleigh waves}

From the theory of wave propagation, the horizontal displacement $u_{x}$ and the vertical displacement $u_{y}$, of a soil particle during the passage of Rayleigh waves can be expressed in the form

$$
u_{x}=A \mathrm{i} k\left[\exp (-a k y)-\frac{1+b^{2}}{2} \exp (-b k y)\right] .
$$

and

$$
u_{y}=A k\left[-a \exp (-a k y)+\frac{1+b^{2}}{2 b} \exp (-b k y)\right] .
$$

If only the real part is considered, we have

$$
\begin{aligned}
& u_{x}=f_{1}(y) \sin [k(x-c t)], \\
& u_{y}=f_{2}(y) \cos [k(x-c t)],
\end{aligned}
$$

where

$$
\begin{gathered}
f_{1}(y)=-A k\left[\exp (-a k y)-\frac{1+b^{2}}{2} \exp (-b k y)\right], \\
f_{2}(y)=A k\left[-a \exp (-a k y)+\frac{1+b^{2}}{2 b} \exp (-b k y)\right] \\
a=\sqrt{1-\frac{c^{2}}{c_{\mathrm{P}}^{2}}} \quad b=\sqrt{1-\frac{c^{2}}{c_{\mathrm{S}}^{2}}}
\end{gathered}
$$

with $k$ being the wave number which can be got from the formulation $k=\omega / c, c_{\mathrm{P}}, c_{\mathrm{S}}$ and $c$ being the dilatational wave velocity, shear wave velocity, and Rayleigh wave velocity, respectively. The parameter $c$ can be got ap- 
proximately from the expression

$$
c=c_{\mathrm{R}} \approx \frac{0.862+1.14 \mu}{1+\mu} c_{\mathrm{S}} .
$$

From equation (5), it can be seen that the trajectory of the soil particle motion describes a retrograde ellipse. The normalized values of horizontal displacement $u_{x} / u_{x 0}$ and vertical displacement $u_{y} / u_{y 0}$ are plotted versus depth in Figure 2.

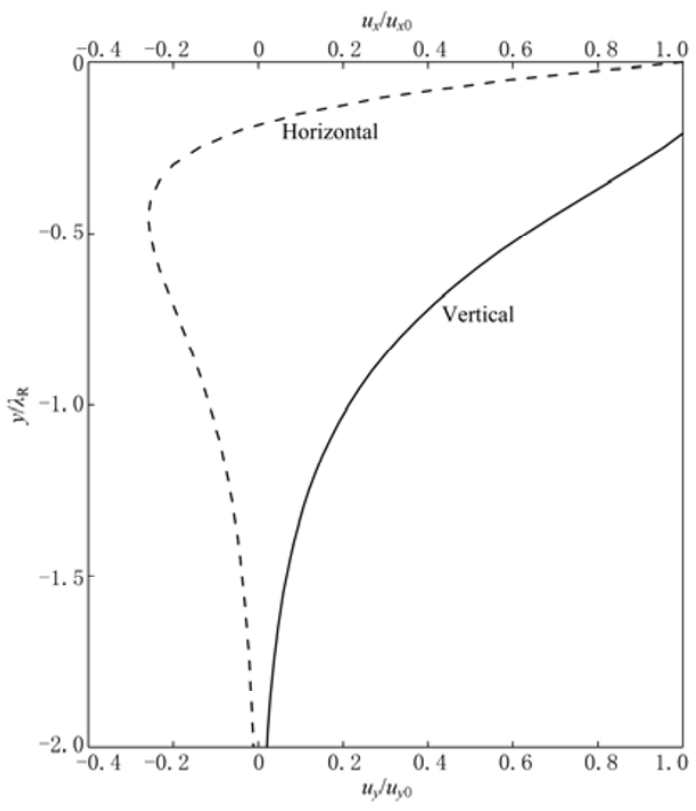

Figure 2 Rayleigh wave displacement variation with depth $(\mu=0.3)$.

\section{Approximate Rayleigh wave field}

The above formulas are applied to the steady waves. In reality most of the wave types are transient waves, such as seismic waves. In the linear medium, transient waves can be seen as the superposition of steady waves. The approximate seismic waves are put forward to simulate Rayleigh wave motion. The method will be described in detail as follows.

Based on the Fourier transform, the approximate Rayleigh waves can be expressed as the superimposition of the harmonious waves. Assume $u_{x}$ and $u_{y}$ are the horizontal and vertical displacement time histories of the earthquake respectively. They can be expressed respectively as

$$
u_{x}=\sum_{n=0}^{\infty} f_{1}^{n}(y) \sin \left[k_{n}(x-c t)\right]
$$

and

$$
u_{y}=\sum_{n=0}^{\infty} f_{2}^{n}(y) \cos \left[k_{n}(x-c t)\right]
$$

in which

$$
\begin{gathered}
f_{1}^{n}(y)=-A_{n}\left[\exp \left(-a k_{n} y\right)-\frac{1+b^{2}}{2} \exp \left(-b k_{n} y\right)\right], \\
f_{2}^{n}(y)= \\
A_{n} k_{n}\left[-a \exp \left(-a k_{n} y\right)+\frac{1+b^{2}}{2 b} \exp \left(-b k_{n} y\right)\right],
\end{gathered}
$$

$k_{n}=\omega_{n} / c, \omega_{n}=\pi n / T, T$ represents the period, and $A_{n}$ is a amplitude parameter, which can be derived from the wave motion. The parameters $a, b$ and $c$ can be got from the aforementioned formula.

Assume one of the horizontal earthquake time histories is known as $h(t)$, which can be considered as incident wave at the origin of the coordinate, that is $x=0$, $y=0$. Substituting $x=0, y=0$ into equations ( $8 \mathrm{a})$ and (9a), $h(t)$ can be expressed as

$$
h(t)=\frac{1-b^{2}}{2} \sum_{n=1}^{\infty} A_{n} \sin \left(\omega_{n} t\right) .
$$

Considering the orthogonality of the trigonometric functions, the amplitude parameter $A_{n}$ can be demonstrated as

$$
A_{n}=\frac{2}{1-b^{2}}\left[\frac{2}{T} \int_{0}^{T} h(t) \sin \left(\omega_{n} t\right) \mathrm{d} t\right] .
$$

Substituting the amplitude parameter $A_{n}$ into equation (9), the two dimensional Rayleigh wave field can be obtained. But the calculation will be time consuming using the method mentioned above. And the stability of numerical integral is not easy to ensure, so the fast Fourier transfer (FFT) method will be a better choice.

The fast Fourier transform (FFT for short) is a computer algorithm for calculating discrete Fourier transform. Suppose there exist some discrete series $\left\{x_{k}\right\}$, $k=0,1,2, \cdots, N-1$. And assume $t_{k}$ is sampling time, where $t_{k}=k \Delta$, and $\Delta$ is sampling interval. The formal definition of the discrete Fourier transform (DFT for short) of the series $\left\{x_{k}\right\}(k=0,1,2, \cdots, N-1)$ is

$$
X_{n}=\sum_{k=0}^{N-1} x_{k} \mathrm{e}^{2 \pi \mathrm{i} k n / N}
$$

The inverse discrete Fourier transform is given by

$$
x_{k}=\frac{1}{N} \sum_{n=0}^{N-1} X_{n} \mathrm{e}^{-2 \pi i k n / N} .
$$

This is usually Fourier transform in the complex domain. In this paper, the Fourier in the real domain will be used, 
that is discrete sinusoidal transfer and discrete cosine transfer.

Considering a discrete signal:

$$
h_{k} \equiv h\left(t_{k}\right) \quad t_{k} \equiv k \Delta \quad k=0,1,2, \cdots, N-1,
$$

where $\Delta$ is the sampling interval, $t_{k}$ is the sampling time; the corresponding sample frequency is $1 / \Delta$.

The discrete Fourier transfer can be expressed as follows:

$$
\left\{\begin{array}{l}
H_{n}=\sum_{k=0}^{N-1} h_{k} \mathrm{e}^{2 \pi \mathrm{i} k n / N} \\
h_{k}=\frac{1}{N} \sum_{n=0}^{N-1} H_{n} \mathrm{e}^{-2 \pi \mathrm{i} k n / N}
\end{array} .\right.
$$

Accordingly, the discrete sinusoidal transfer can be expressed as

$$
\left\{\begin{array}{l}
H_{n}=\sum_{k=0}^{N-1} h_{k} \sin (\pi \mathrm{i} k n / N) \\
h_{k}=\frac{2}{N} \sum_{n=0}^{N-1} H_{n} \sin (\pi \mathrm{i} k n / N)
\end{array} .\right.
$$

And the discrete cosine transfer is

$$
\left\{\begin{array}{l}
H_{n}=\sum_{k=0}^{N-1} h_{k} \cos (\pi \mathrm{i} k n / N) \\
h_{k}=\frac{2}{N} \sum_{n=0}^{N-1} H_{n} \cos (\pi \mathrm{i} k n / N)
\end{array} .\right.
$$

The horizontal earthquake time history $h(t)$ can be expressed by equation (14). Substituting it into equation (11), the amplitude parameter can be obtained as follows:

$$
\begin{gathered}
A_{n}=\frac{2}{1-b^{2}}\left[\frac{2}{T} \int_{0}^{T} h(t) \sin \left(\omega_{n} t\right) \mathrm{d} t\right]= \\
\frac{2}{1-b^{2}} \frac{2}{N}\left[\sum_{k=0}^{N-1} h_{k} \sin (\pi \mathrm{i} k n / N)\right] .
\end{gathered}
$$

According to equation (16), the part in the bracket can be obtained using the fast discrete sinusoidal transfer (FDST). Substituting equation (18) into equation (9), the functions that describe the variation along the depth can be obtained.

$$
\begin{gathered}
f_{1}^{n}(y)=-\frac{2}{1-b^{2}} \frac{2}{N}\left[\sum_{k=0}^{N-1} h_{k} \sin (\pi \mathrm{i} k n / N)\right] \\
{\left[\exp \left(-\frac{a y}{c} \omega_{n}\right)-\frac{1+b^{2}}{2} \exp \left(-\frac{b y}{c} \omega_{n}\right)\right]} \\
f_{2}^{n}(y)=\frac{2}{1-b^{2}} \frac{2}{N}\left[\sum_{k=0}^{N-1} h_{k} \sin (\pi \mathrm{i} k n / N)\right] .
\end{gathered}
$$

$$
\left[-a \exp \left(-\frac{a y}{c} \omega_{n}\right)+\frac{1+b^{2}}{2 b} \exp \left(-\frac{b y}{c} \omega_{n}\right)\right] \frac{\omega_{n}}{c}
$$

For the arbitrary node $(x, y)$, the displacement can be expressed as

$$
\begin{gathered}
u_{x}=\sum_{n=0}^{N-1} f_{1}^{n} \sin \left(\omega_{n} \frac{x}{c}\right) \cos \left(\omega_{n} t\right)- \\
\sum_{n=0}^{N-1} f_{1}^{n} \cos \left(\omega_{n} \frac{x}{c}\right) \sin \left(\omega_{n} t\right)
\end{gathered}
$$

and

$$
\begin{gathered}
u_{y}=\sum_{n=0}^{N-1} f_{2}^{n} \cos \left(\omega_{n} \frac{x}{c}\right) \cos \left(\omega_{n} t\right)+ \\
\sum_{n=0}^{N-1} f_{2}^{n} \sin \left(\omega_{n} \frac{x}{c}\right) \sin \left(\omega_{n} t\right) .
\end{gathered}
$$

We continue to consider displacements of the whole wave field and proceed to show that, when the displacements at the origin of the coordinate $x=0$ are known, we can calculate the wave motion at any time under the concept of wave propagation. This can be illustrated as follows:

$$
\begin{gathered}
u_{x}(0, y ; t)=-\sum_{n=0}^{N-1} f_{1}^{n} \sin \left(\omega_{n} t\right)=\frac{2}{1-b^{2}} . \\
\frac{2}{N} \sum_{n=0}^{N-1}\left\{[ \sum _ { k = 0 } ^ { N - 1 } h _ { k } \operatorname { s i n } ( \frac { \pi \mathrm { i } k n } { N } ) ] \left[\exp \left(-\frac{\pi a y}{T c} n\right)-\right.\right. \\
\left.\left.\frac{1+b^{2}}{2} \exp \left(-\frac{\pi b y}{T c} n\right)\right] \sin \left(\frac{\pi \mathrm{i} k n}{N}\right)\right\}, \\
\frac{2}{N} \sum_{n=0}^{N-1}\left\{[ \sum _ { k = 0 } ^ { N - 1 } h _ { k } \operatorname { s i n } ( \frac { \pi \mathrm { i } k n } { N } ) ] \left[-a \exp \left(-\frac{\pi a y}{T c} n\right)+\right.\right. \\
\left.\left.\frac{1+b^{2}}{2} \exp \left(-\frac{\pi b y}{T c} n\right)\right] \frac{\pi n}{T c} \cos \left(\frac{\pi \mathrm{i} k n}{N}\right)\right\} .
\end{gathered}
$$

Equation (21a) can be resolved with the inverse fast discrete sinusoidal transfer (iFDST) and Equation (21b) with the inverse fast discrete cosine transfer (iFDCT). The displacement time history of any arbitrary node can be obtained using the wave propagation method considering time delay.

$$
u_{x}(x, y ; t)= \begin{cases}0 & t-\frac{x}{c}<0 \\ u_{x}\left[0, y ; t-\frac{x}{c}\right] & t-\frac{x}{c} \geq 0\end{cases}
$$




$$
u_{y}(x, y ; t)=\left\{\begin{array}{lc}
0 & t-\frac{x}{c}<0 \\
u_{y}\left[0, y ; t-\frac{x}{c}\right] & t-\frac{x}{c} \geq 0
\end{array}\right.
$$

Now we see that the whole Rayleigh wave field has been achieved to approximate the earthquake. The procedure is expressed in Figure 3. As an example, the displacement time history of east-west component of the well known El-Centro wave record is shown in Figure 4.

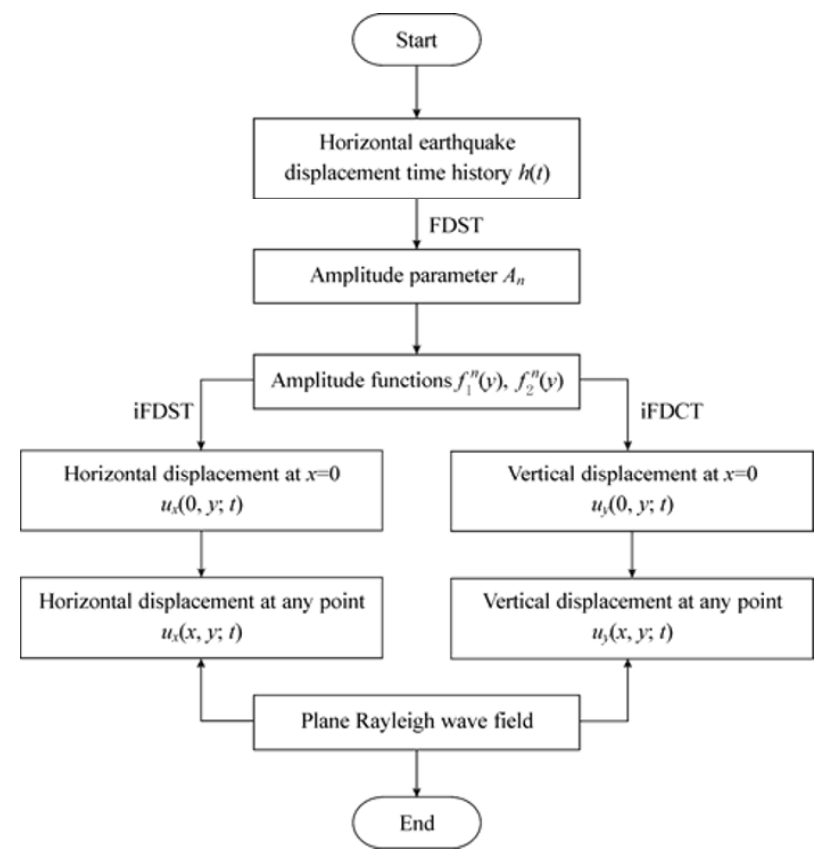

Figure 3 Basic generation procedure of the plane Rayleigh wave field.

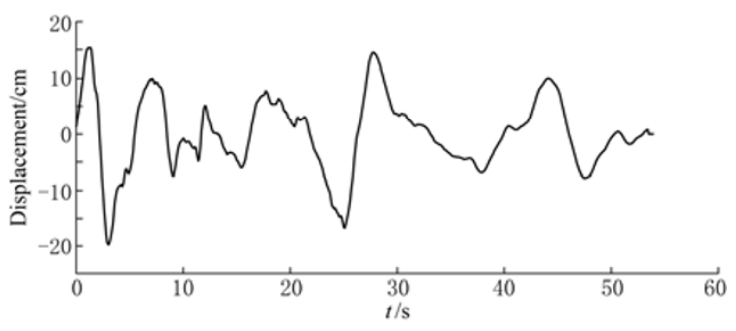

Figure 4 Displacement time history of east-west component of El-Centro wave.

Using the method mentioned above, the calculated vertical displacement time history is depicted in Figure 5. Equation (6) expresses the variation of the Rayleigh amplitude along the soil depth. So the displacement time history input can also be gotten at different depths.

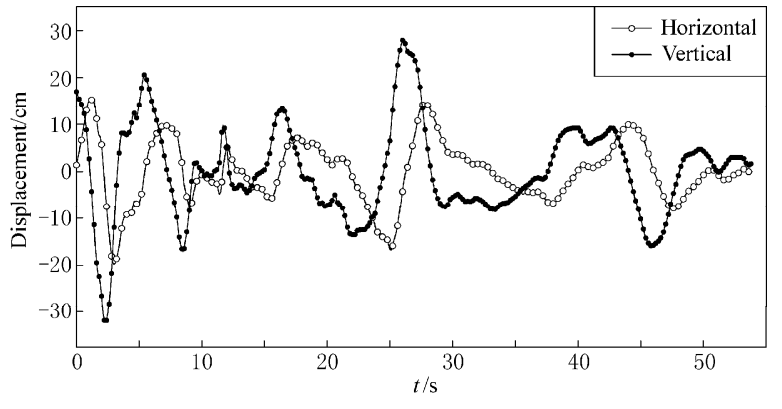

Figure 5 Horizontal and vertical displacement time histories at the free surface.

\section{Free field analyses during the passage of Rayleigh waves}

In order to verify effectiveness and feasibility of the proposed Rayleigh wave's excitation method, several numerical examples are analyzed in this section. A free field analysis under the Rayleigh waves is considered. Two dimensional plane strain elements are chosen in the finite element analysis. Figure 6 is the sketch of the finite element model. Considering the characteristic of the finite element formulation of wave motion, the mesh size is set as $1 \mathrm{~m} \times 1 \mathrm{~m}$. In order to reduce the reflection and dispersion of the wave on the boundary, the infinite elements at the boundaries are adopted.

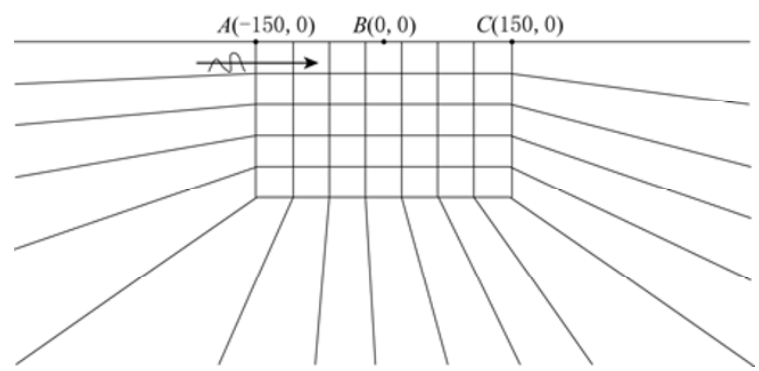

Figure 6 Sketch of the analysis model.

The soil parameters used in calculation are as follows: mass density is $1.8 \times 10^{3} \mathrm{~kg} / \mathrm{m}^{3}$, elastic modulus is $20 \mathrm{MPa}$ and Poisson's ratio is 0.3 . From the parameters above, we can get the Rayleigh wave velocity $c=c_{\mathrm{R}}=$ $0.926, c_{\mathrm{S}}=60.5604, \lambda_{\mathrm{R}}=c_{\mathrm{R}} / f=20.1868 \mathrm{~m}, a=0.86886$, $b=0.37715, k=0.3112$.

The horizontal and vertical displacements of Rayleigh wave are input from the left side. The excitation displacement is deduced from equation (5) and the content $A=1$ is assumed. The excitation displacement time history is multiplied by a Hanning window function $u(t)$ in order to express the wave propagation more 
clearly.

$$
u(t)= \begin{cases}\frac{1}{2}-\frac{1}{2} \cos \frac{2 \pi t}{T} & 0 \leq t \leq 1 \\ 0 & 1<t \leq 1\end{cases}
$$

After operation, the horizontal and vertical displacement time histories at the free surface are shown in Figure 7 respectively.

It is well known that the passage of the Rayleigh waves is the retrograde ellipse. Figure 8 is contour plot of von Mises stress of soil during the wave propagation at the representative moment. It is obvious that the deformation of the soil is just like an adverse ellipse, when the waves propagate to the boundary, the wave motion is well absorbed.

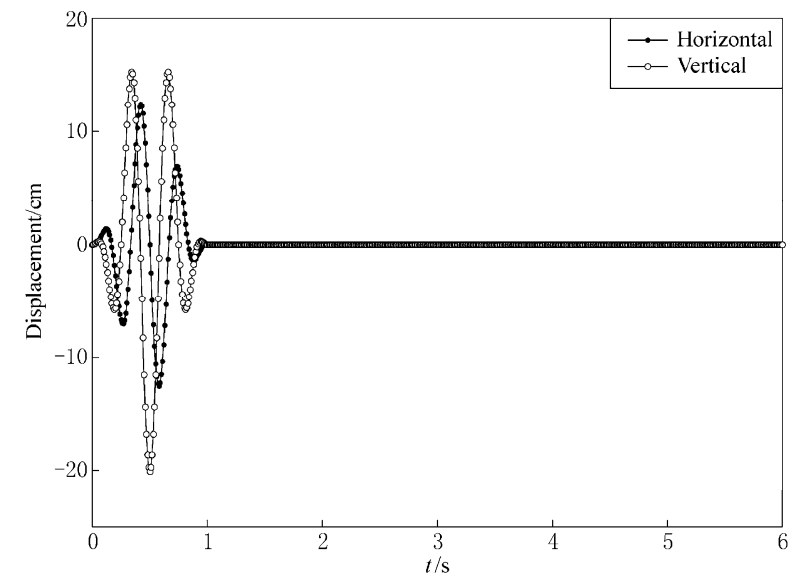

Figure 7 Input time history at the free surface.

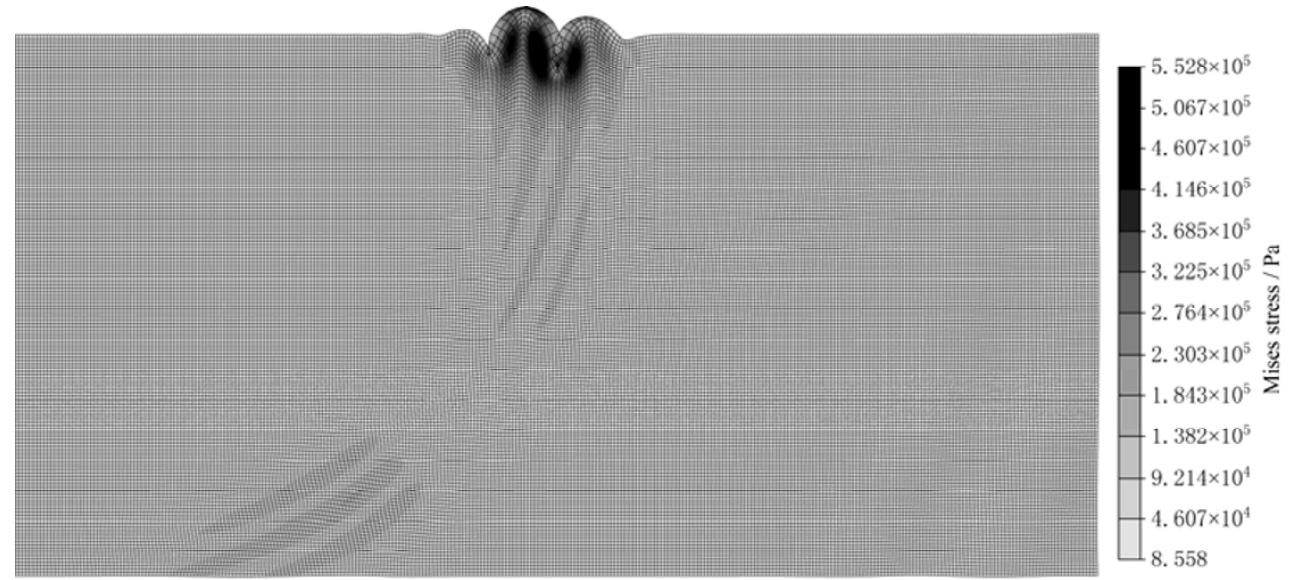

(a)

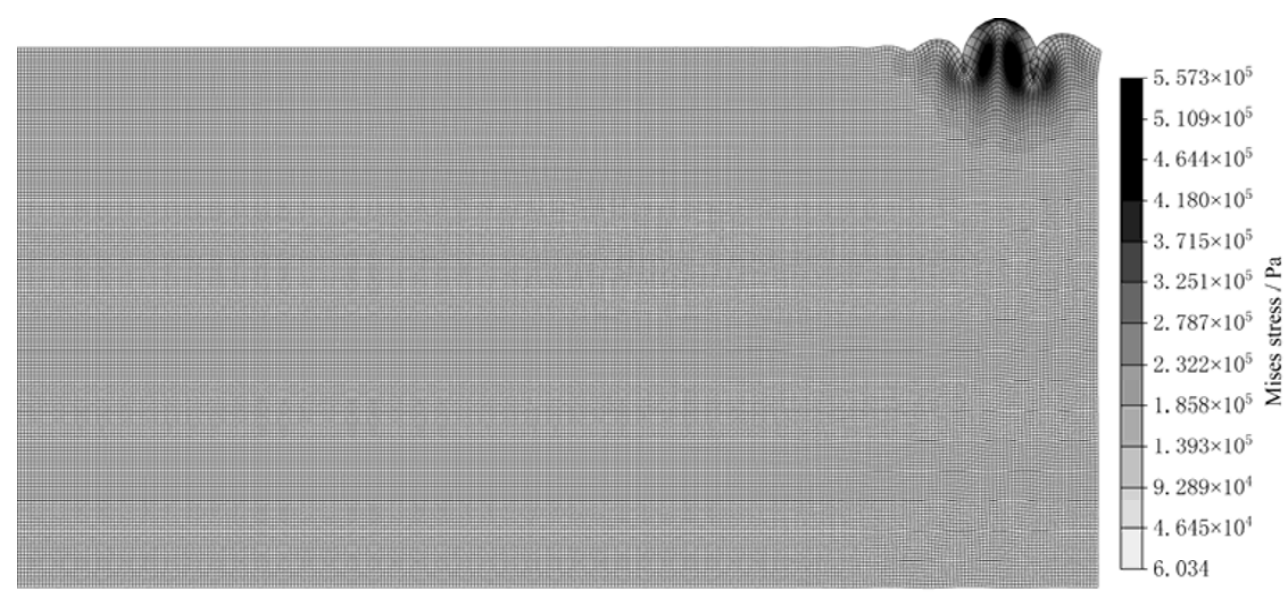

(b)

Figure 8 Von Mises stress during the passage of Rayleigh waves (scale factor 50) at $3.0 \mathrm{~s} \mathrm{(a)} \mathrm{and} 5.2 \mathrm{~s}$ (b).

The analytical solutions are compared with numerical solutions to verify the precision of the simulation. Take the node $B$ as example. The node $B$ is at the center of the free surface as shown in Figure 6. Figure 9 is the displacement comparison between the numerical and analytical results of horizontal and vertical displacements. From Figure 9, it can be seen that the analytical solution and numerical solution agree well. The time delay can also be seen in the figure. 

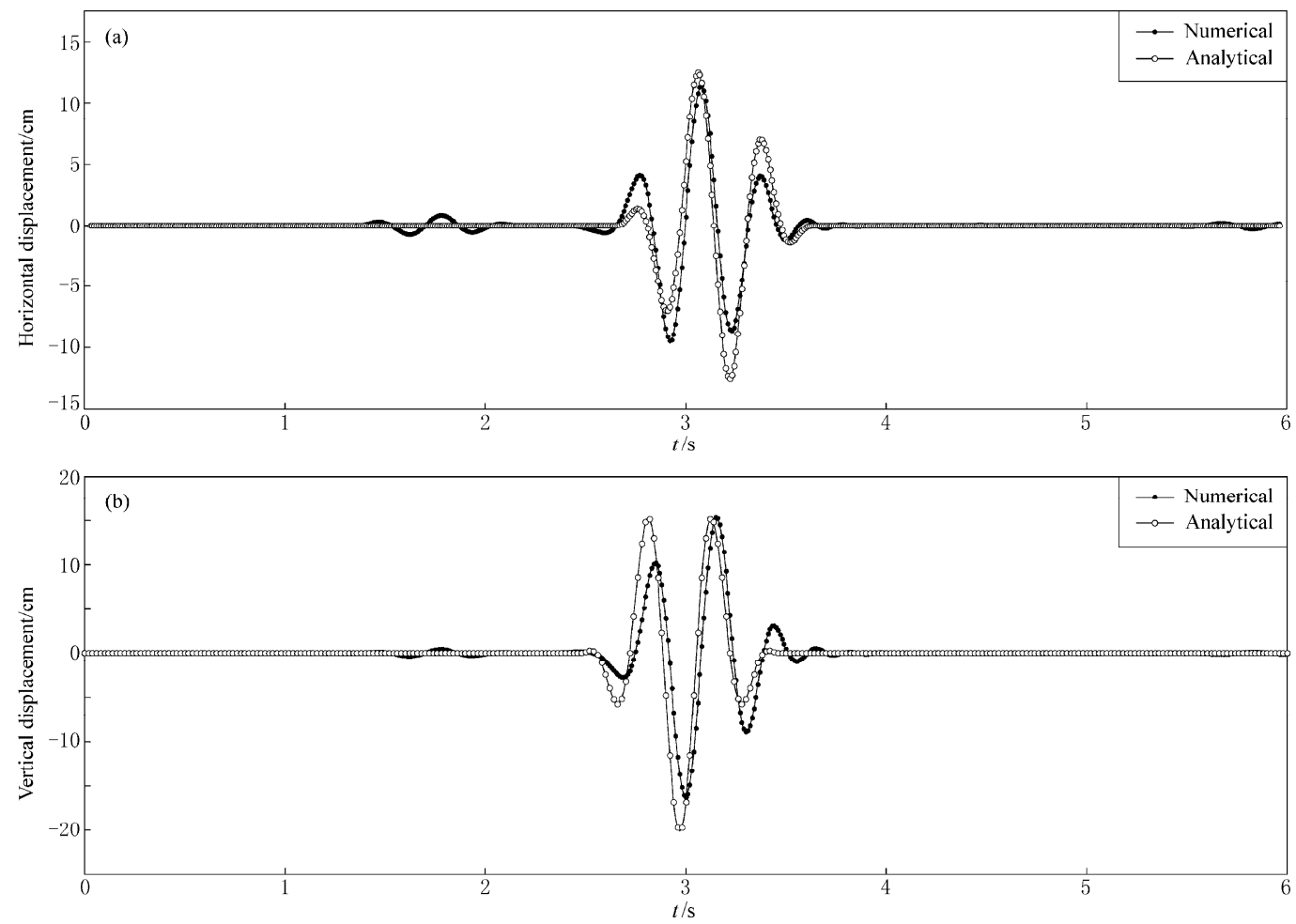

Figure 9 Horizontal (a) and vertical (b) displacement time histories of the node $B$ in Figure 6.

\section{Utility tunnel response during the passage of the Rayleigh waves}

Utility tunnel is typically shallow buried underground structures, and Rayleigh wave has the character of large amplitude and much energy near the surface. So Rayleigh wave will be the key factor to control the response.

Total size of the calculation model is $60 \mathrm{~m} \times 30 \mathrm{~m} \times$ $100 \mathrm{~m}$, in which the structure model is of $3 \mathrm{~m} \times 3 \mathrm{~m} \times 100$ $\mathrm{m}$ with $3 \mathrm{~m}$ buried depth. The section for structure is rectangular, with the wall thickness of the top and side slab are $0.3 \mathrm{~m}$ and the bottom slab is $0.4 \mathrm{~m}$. Figure 10 is the three dimensional finite element model of the soil-structure and the infinite elements are added to simulate the artificial boundary condition. In order to display the model expressly, the infinite elements are omitted here.

The Drucker-Prager model (Huo, 2005) is adopted in simulation of the soil. The structure is assumed as elastic, which is reasonable for the underground structure. Table 1 lists the material parameters of soil and structure. The slippage and the separation between soil and structure are not considered here for the time being.

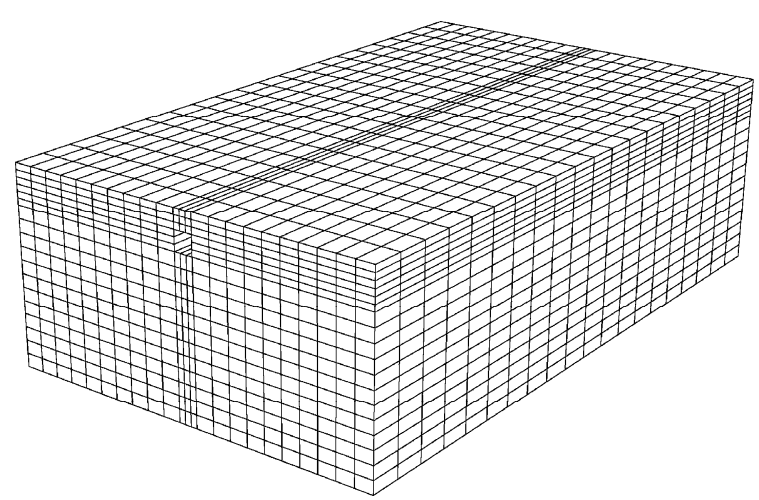

Figure 10 The finite element model for numerical calculation.

Table 1 Material parameters of the soil and structure

\begin{tabular}{ccccc}
\hline & $\begin{array}{c}\text { Unit weight } \\
/ \mathrm{kN} \cdot \mathrm{m}^{-3}\end{array}$ & $\mu$ & $\begin{array}{c}E \\
/ \mathrm{MPa}\end{array}$ & $k$ \\
\hline Soil & 19.6 & 0.30 & 20 & 0.8 \\
Structure & 24.0 & 0.15 & 26400 & \\
\hline \hline & Friction angel & Dilation angel & Yield stress & \\
& $/{ }^{\circ}$ & 3 & 500 & \\
\hline Soil & 30 & & & \\
Structure & & $3 \mathrm{kPa}$ & \\
\hline
\end{tabular}

Note: $k$ is the ratio of the flow stress in tri-axial tension to the flow stress in tri-axial compression, $\mu$ is Poisson's ratio, and $E$ is Elastic modulus. 
According to the above technique about the generation of the approximate Rayleigh wave field, the horizontal and vertical displacement time histories at the top boundary are shown in Figure 11. The input displacement along the depth is obtained according to equation (9). The approximate Rayleigh wave excitation is input from the left side of the model.

After calculations using finite element software ABAQUS, the results are as follows. Figure 12 is the von Mises stress contour during the Rayleigh wave propagation in soil. We can see the wave front clearly and the waves propagate stably forward. Figure 13 is the structural von Mises stress contour at representative time, from which we can see the deformation of structure
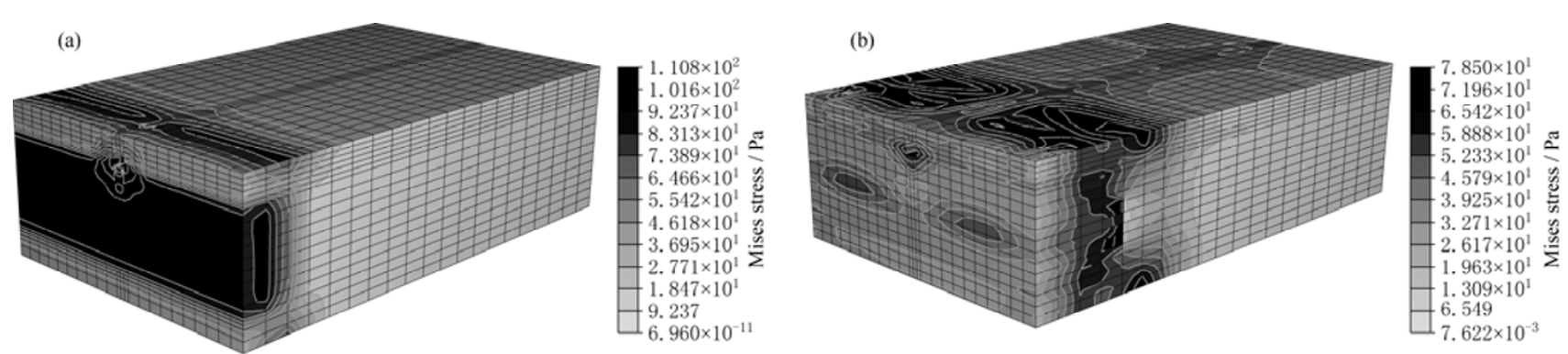

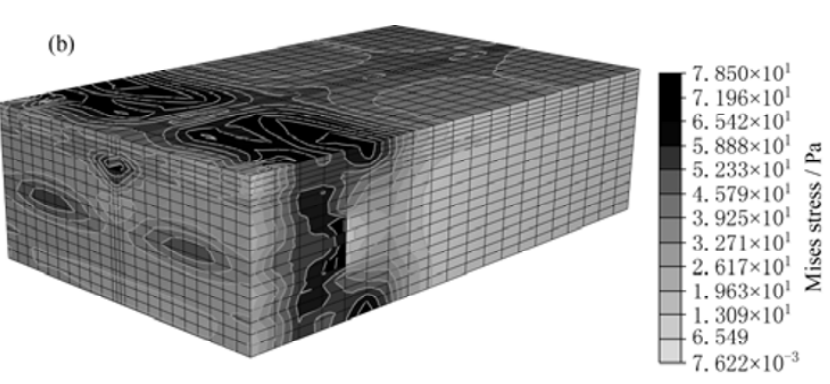

is mainly the bending deflection, which will be clearly demonstrated from the following strain time history. In order to express them clearly, a scale factor of 200 is adopted.

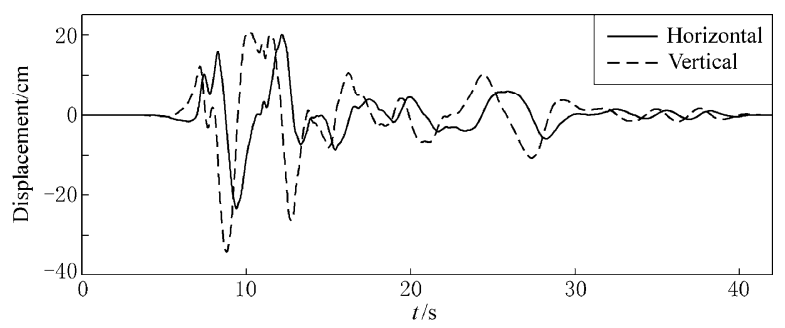

Figure 11 Horizontal and vertical Rayleigh wave input time histories at the surface of the model.

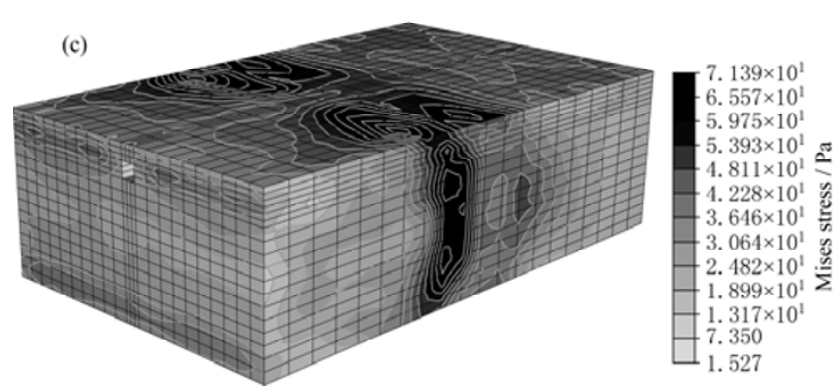

Figure 12 Wave propagation in soil at representative time. (a) Von Mises stress contour at $0.1 \mathrm{~s}$; (b) Von Mises stress contour at $0.3 \mathrm{~s}$; (c) Von Mises stress contour at $0.8 \mathrm{~s}$.
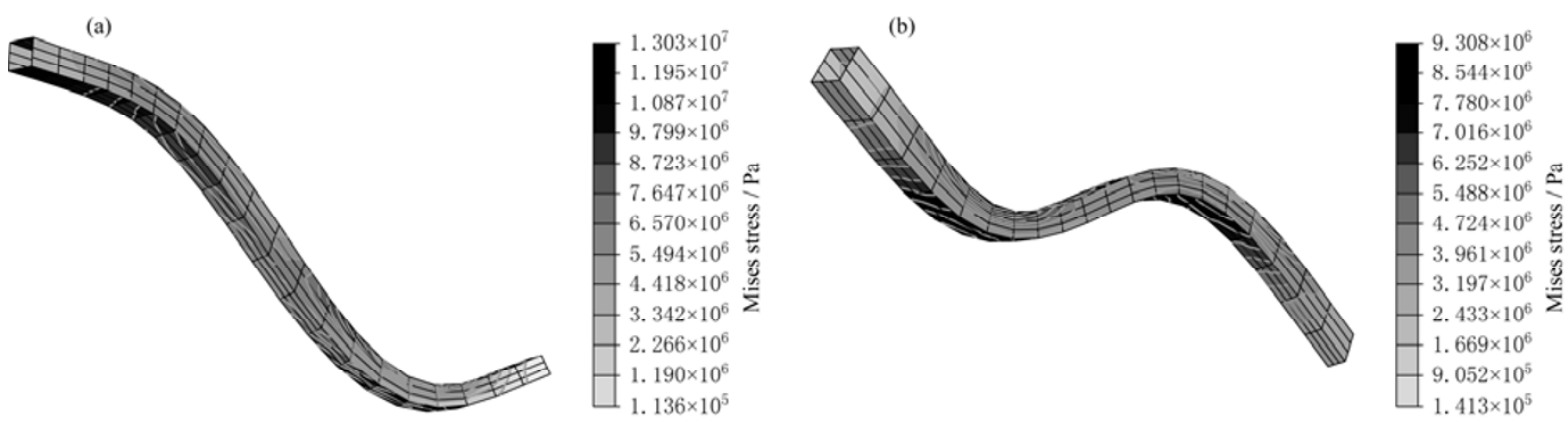

Figure 13 Von Mises stress contour and deformation of structure at $10.8 \mathrm{~s}$ (a) and $11.5 \mathrm{~s} \mathrm{(b)}$ with scale factor of 200.

The above analysis is from the aspect of the whole deformation of the soil and structure. In order to study the structural response systematically, the strain time history of the utility tunnel is investigated. Figure 14 is 
the sketch of output points on the utility tunnel.

The strain time history (Figure 15) of the corresponding top and bottom point of the utility tunnel shows that the signal of the strain on the top points and the corresponding bottom points are opposite, indicating that the structural deformation is mainly the global bending deflection. On the other hand, the amplitude of the strain on the top of the structure is about twice as much as that at bottom, which agrees with the characteristic of the Rayleigh waves. Figure 16 is the stain time history of points $A_{1}$ and $A_{5}$. Time delay between the two points can be clearly observed. As shown in the figure, the time delay is about 1.15 seconds and the distance between the points $A_{1}$ and $A_{5}$ is 65 meters. So the

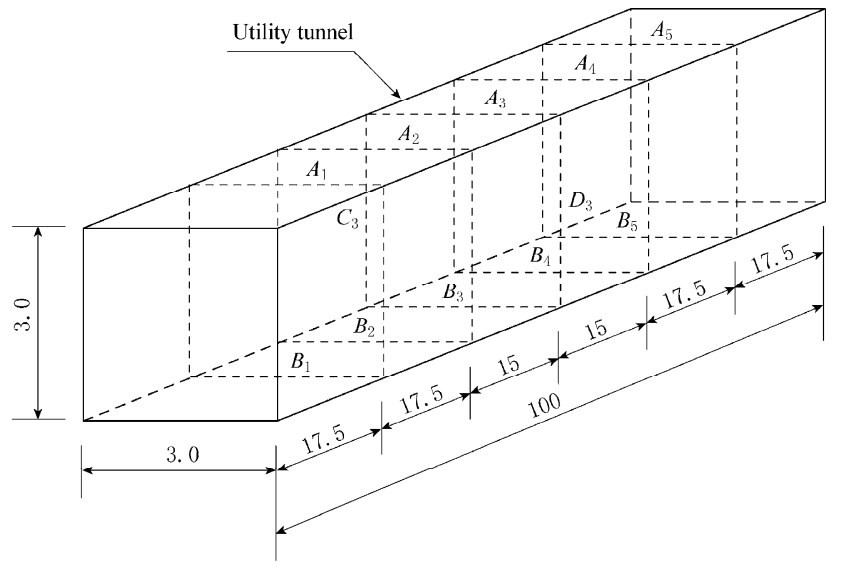

Figure 14 Typical output point on the utility tunnel.

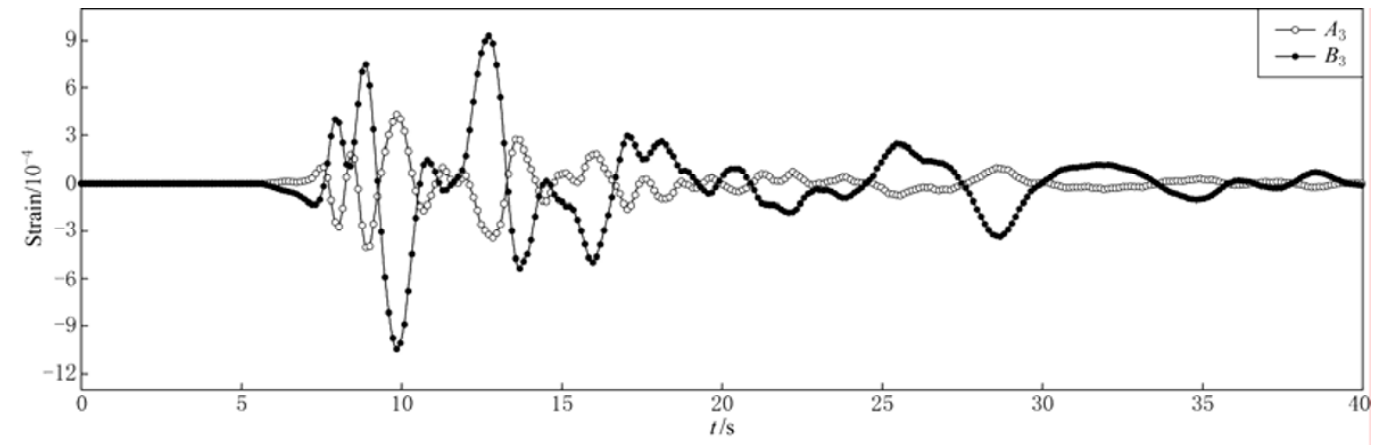

Figure 15 Strain time histories of the points $A_{3}$ and $B_{3}$.

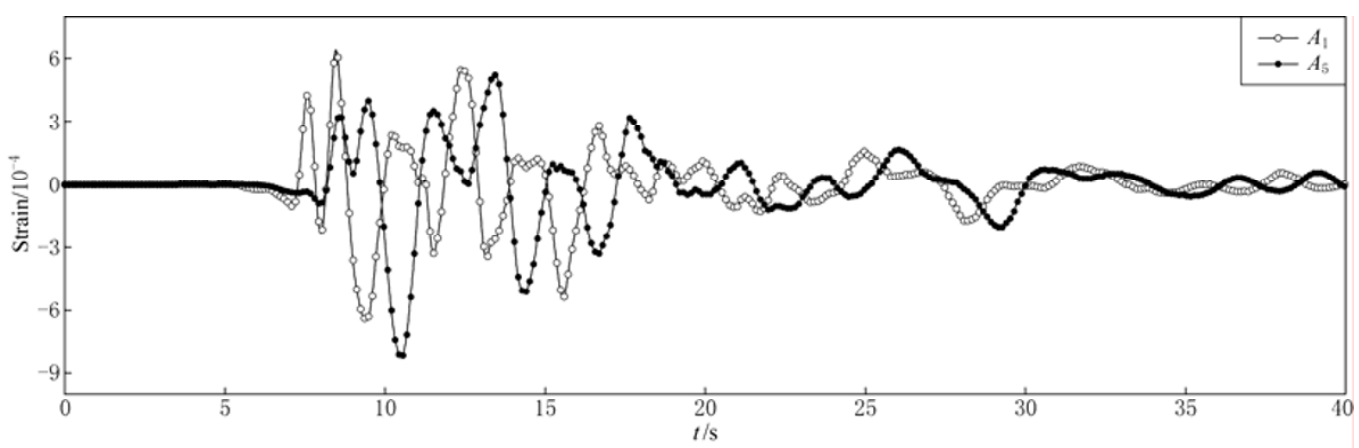

Figure 16 Strain time histories of the points $A_{1}$ and $A_{5}$.

wave group velocity is about $56.5 \mathrm{~m} / \mathrm{s}$. On the other hand, from soil parameters, the shear wave velocity can be deduced from the formula $c_{\mathrm{S}}=(G / \rho)^{1 / 2}$ and then using the relation between the shear velocity and Rayleigh wave velocity, the Rayleigh wave velocity can be approximately estimated as $60.5 \mathrm{~m} / \mathrm{s}$. The two velocities are close, which indicates that the wave is stable during the propagation.

In order to further verify the interaction between the soil and the structure, the interface between the soil and the structure is considered as Coulomb friction, and the friction coefficient is $\mu=0.4$. Figure 17 is comparison of the strain time history of the point $A_{1}$ in the case of no sliding and sliding. Table 2 is the strain amplitude of different output points. From these results, we can see that the strain amplitudes decrease when the sliding is considered. This is because that when no sliding is considered, soil constricts the deformation of the structure. So the strain of the structure is increased. 


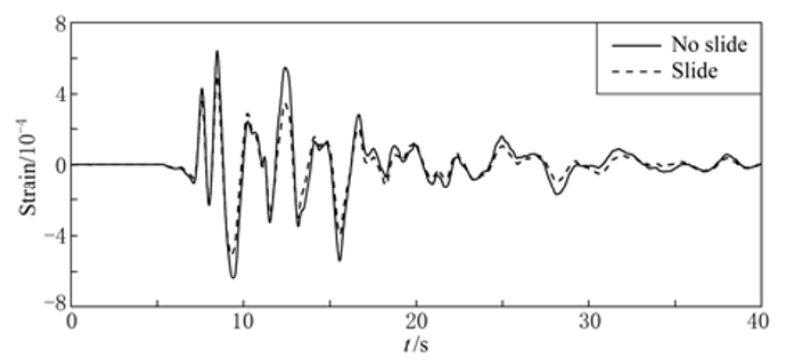

Figure 17 Strain time history of point $A_{1}$ in the case of sliding and no sliding between the soil and structure.

Table 2 Strain amplitude of structure under the different interface conditions

\begin{tabular}{ccc}
\hline \multirow{2}{*}{ Output point } & \multicolumn{2}{c}{ Strain amplitude of structure $/ 10^{-6}$} \\
\cline { 2 - 3 } & $\mu=0.4$ & $\mu=\infty$ \\
\hline$A_{1}$ & -509.97 & -638.85 \\
$A_{2}$ & -791.72 & -1070 \\
$A_{3}$ & -848.61 & -1200 \\
$A_{4}$ & -891.26 & -1170 \\
$A_{5}$ & -641.73 & -827.43 \\
\hline
\end{tabular}

From the property of the Rayleigh wave, its amplitude decreases with depth. So the different buried depth of the structure is considered here. The results of buried depth $9 \mathrm{~m}$ and $15 \mathrm{~m}$ are presented. Figure 18 is the strain time history of the point $A_{3}$ under different buried depth. Table 3 is the strain amplitude of output points. From Figure 18 and Table 2, it is obvious that the strain amplitude of top plate of the structure decreases with the buried depth, which is accord with the property of Rayleigh waves; while the strain amplitude of bottom plate of the structure increases with buried depth. The reason for this is that because the base boundary of the model is considered as the base rock, and the artificial boundary no considered here. So the wave reflection affects the results of the bottom plate and the further research is needed.

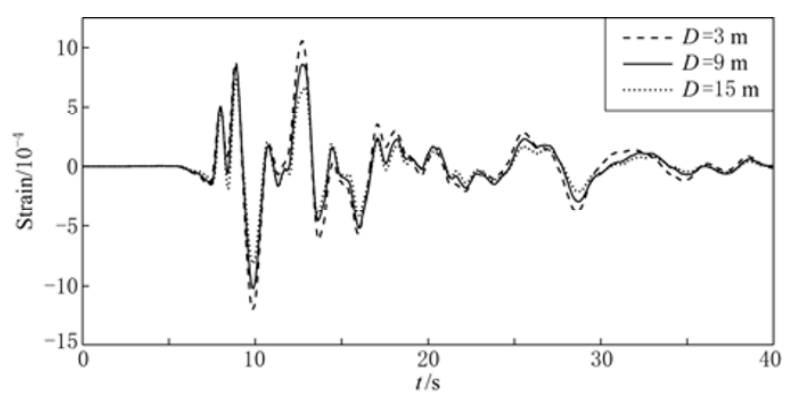

Figure 18 Strain time history of the point $A_{3}$ with different buried depth $(D)$.
Table 3 Strain amplitude of structure under different buried depth $D$

\begin{tabular}{cccc}
\hline \multirow{2}{*}{ Output points } & \multicolumn{3}{c}{ Strain amplitude $/ 10^{-6}$} \\
\cline { 2 - 4 } & $D=3 \mathrm{~m}$ & $D=9 \mathrm{~m}$ & $D=15 \mathrm{~m}$ \\
\hline$A_{1}$ & 641.01 & 601.60 & -533.66 \\
$A_{2}$ & -1070 & -920.85 & -759.58 \\
$A_{3}$ & -1200 & -1020 & -814.45 \\
$A_{4}$ & -1170 & -902.21 & -914.21 \\
$A_{5}$ & -827.43 & -818.28 & -720.81 \\
$B_{1}$ & 245.45 & 325.53 & -408.02 \\
$B_{2}$ & 299.32 & 445.01 & 602.92 \\
$B_{3}$ & -261.93 & 438.19 & 656.16 \\
$B_{4}$ & -379.65 & 506.12 & 668.42 \\
$B_{5}$ & 261.38 & 411.31 & 511.54 \\
\hline
\end{tabular}

\section{Discussion and conclusions}

The effect of surface waves especially the Rayleigh waves is of great importance for seismic assessment of shallow buried underground structures like utility tunnel. Taking utility tunnel as an example, this paper investigates soil displacement caused by the passage of Rayleigh waves.

On the basis of the theory of wave motion, the steady Rayleigh waves are studied and the response of the free field is examined. From the stress map of soil, the adverse ellipse propagating of the soil can be seen clearly and the analytical results agree well with the numerical ones, which indicates that the proposed wave excitation method is proper for Rayleigh wave. The wave can propagate steadily in soil.

The approximate Rayleigh wave is put forward in the paper and the Rayleigh wave field is obtained via FFT techniques in the real domain. A three dimensional finite element model is adopted in this paper to calculate the response of the utility tunnel. The results show that the deformation of the structure is generally greater under the passage of Rayleigh waves. The bending deflection is the most common deformation, and the deformation at the top of the structure is about twice as much as that at the bottom, which agrees with the character of the Rayleigh waves. The effect of soil-structure interface and the buried depth of underground structure are also investigated via parameter analysis. The results demonstrate that the strain amplitude of the structure will be decreased when sliding is considered. Moreover, the response of the structure will decrease with increasing buried depth of the structure.

In general, the response character of the structure is different under Rayleigh waves. And this work just is a start. There is still much work to do in the future. 
Acknowledgements This paper is jointly supported by key project of the National Science and Technology Pillar Program (Grant No. 2006BAJ03B03) and Research Fund from State Key Laboratory for Disaster Reduction in Civil Engineering (Grand No. SLDRCE08-C-03).

\section{References}

Alterman Z and Kara F C (1968). Propagation of elastic waves in layered media by finite difference methods. Bull Seism Soc Amer 58(1): 367-398.

Clayton R and Engquist B (1977). Absorbing boundary conditions for acoustic and elastic wave equations. Bull Seism Soc Amer 67(6): 1 529-1 540.

Hashash Y M A, Hook J J, Schmidt B and Yao J I-Chiang (2001). Seismic design and analysis of underground structures. Tunneling and Underground Space Technology 16: 247-293.

Huo H (2005). Seismic Design and Analysis of Rectangular Underground Structures. Purdue University, USA, 138-141, 301-334.

Ismail I M and Mullen C (2000). Soil structure interaction issues for three dimensional computational simulations of nonlinear seismic response. ASCE 14th Engineering Mechanics Conference (EM2000). Austin, TX, 21-24 May 2000.

Liao Z P (2002). Introduction to Engineering Wave Motion Theories for Engineering. Second edition. Science Press, Beijing, 215-217 (in Chinese).

Liao Z P, Wong H L, Yang B and Yuan Y (1984). A transmitting boundary for transient wave analyses. Scientia Sinica (Series A) 27(10): $1063-1076$.

Lysmer J and Kuhlemeyer R L (1969). Finite dynamic model for infinite media. J Eng Mech, ASCE 95(4): 859-877.

Makris N (1994). Soil-pile interaction during the passage of Rayleigh waves: an analytical solution. Earthq Eng Struct Dynam 23: 153-167.
Makris N and Badoni D (1995). Seismic response of pile groups under oblique-shear and Rayleigh waves. Earthq Eng Struct Dynam 24: 517-532.

Matsui J, Ohtomo K, Kawai I and Okaichi A (2001). Research on streamlining seismic safety evaluation of underground reinforced concrete duct-type station - Part-3. Analytical simulation by RC Macro-model and simple soil model. Transactions, SMiRT 16. Kluwer Academic Publishers, Washington D.C., Paper No. 1296.

Miller G F and Pursey H (1955). On the partition of energy between elastic waves in a semi-infinite solid. Proc Royal Society, London A 233: 55-69.

Penzien J (2000). Seismically induced racking of tunnel linings. Earthq Eng Struct Dynam 29: 683-691.

Penzien J and Wu C L (1998). Stresses in linings of bored tunnels. Earthq Eng Struct Dynam 27: 283-300.

Prasad S K, Towhata I, Chandradhara G P and Nanjundaswamy P (2004). Shaking table tests in earthquake geotechnical engineering. Current Science 87(10): 1398-1 404.

Smith W D (1974). A nonreflecting plane boundary for wave propagation problems. J Comp Phys 15(4): 492-503.

Wang Jaw-Nan (1993). Seismic Design of Tunnels - A Simple State-of-the-Art Design Approach. Parsons Brinckerhoff Inc., New York, 87-134.

Yue Q X, Shi X J and Feng M G (2006). Analysis and comparisons of numerical simulations for infinite medium using artificial boundary. Proceedings of the 7th China Conference on Earthquake Engineering. Seismological Press, Beijing, 356-362 (in Chinese).

Zienkiewicz O C, Bettess P, Chinm T C and Emson C (1981). Numerical methods for unbounded field problems and a new infinite element formulation. In: Kalinowski A J ed. Computational Methods for Infinite Domain Media-Structure Interaction. The American Society of Mechanical Engineers, New York, 115-148. 